\title{
Die Krankheitserfinder
}

\author{
Jörg Blech
}

Psychotherapie-Wissenschaft 8 (1) 19-21 2018

www.psychotherapie-wissenschaft.info

CC BY-NC-ND

DOI: $10.30820 / 8242.03$

\begin{abstract}
Zusammenfassung: Die Pharmaindustrie definiert die Gesundheit des Menschen gegenwärtig neu, sodass Gesundheit ein Zustand ist, den keiner mehr erreichen kann. Viele normale Prozesse des Lebens - Geburt, Alter, Sexualität, NichtGlücklichsein und Tod - sowie normale Verhaltensweisen werden systematisch als krankhaft dargestellt. Global operierende Konzerne sponsern die Erfindung ganzer Krankheiten und Behandlungsmethoden und schaffen so ihren Produkten neue Märkte. Denn indem man zappelnde Kinder medikamentös ruhigstellt, Cholesterin zum Risikofaktor Nummer eins erklärt oder fragwürdige Vorsorgeuntersuchungen einführt, kann man viel Geld verdienen.
\end{abstract}

Schlüsselwörter: Gesundheit, Pharmaindustrie, Krankheitserfinder, Medikalisierung

Schon viele Frauen haben sich gefragt, was mit ihrem Mann los ist. Der einst so vitale Partner wird immer dicker. Nachts liegt er grübelnd wach, tagsüber ist er gereizt. Lust auf Sex hat er auch nicht mehr. Lässt der Kerl sich hängen? Oder liegt es daran, dass sein Körper nicht mehr ausreichend Männlichkeitshormone produziert? Blättert man in der Broschüre einer Pharmafirma, scheint die Sache klar. «In Deutschland leiden immer mehr Männer an Testosteron-Mangel. Fast jeder Vierte ab dem 60. Lebensjahr ist davon betroffen", sagen Mitarbeiter des Unternehmens Dr. Kade/Besins in Berlin. Sogar «Männer in jüngeren Jahren können von einem zu Beschwerden führenden Testosteron-Mangel betroffen sein.» Wie es der Zufall will, haben die besorgten Pharmaleute ein Gegenmittel im Angebot: ein Testosteron-Gel, das man sich auf den Bauch schmieren kann.

Die Wechseljahre des Mannes sind ein Lehrstück für eine erfundene Krankheit. Mitarbeiter pharmazeutischer Firmen und Ärzte deuten natürliche Wechselfälle und normale Verhaltensweisen systematisch als krankhaft um. Sie medikalisieren unser Leben. Früher versuchte man, gegen jede Krankheit einen Wirkstoff zu finden. Heute läuft es oftmals umgekehrt - für jeden Wirkstoff wird nach einer Krankheit gesucht.

Kurz bevor das Testosteron-Gel vor einigen Jahren auf den Markt kam, verbreitete eine PR-Firma die Meldung, dass zwei Drittel der befragten Männer «klimakterische Beschwerden hätten: «Dass ein Testosteron-Defizit hierbei ursächlich sein kann, ist mehrheitlich allerdings unbekannt.» Hinter der Meldung stand Kade/Besins - das erfuhr man allerdings nicht. Ein anderer Hersteller, Jenapharm, streute die Botschaft, dass man von «mindestens 2,8 Millionen Betroffenen in Deutschland» ausgehen müsse.

Die Deutsche Gesellschaft für Urologie stellte klar: Männer kommen gar nicht in die Wechseljahre. Tatsächlich ist das Nachlassen der Testosteron-Produktion eine normale Begleiterscheinung des Alters und nur selten Auslöser der beschriebenen Symptome. Der Lebensstil - zum
Beispiel Rauchen, Alkoholkonsum und Bewegung - spielt eine grössere Rolle.

Dennoch läuft das Testosteron-Gel-Geschäft wie geschmiert, die Zahl der Rezepte in Deutschland hat sich mehr als verdreifacht. Das ist nicht ohne Risiko. Denn mehr als 40 Prozent der Männer im Alter von über 50 Jahren haben in der Vorsteherdrüse (Prostata) kleine, schlummernde Krebsherde. Und ausgerechnet diese harmlosen Krebszellen können durch künstlich zugeführtes Testosteron zum Wachsen angeregt werden. Den Wechseljahren des Mannes können viele andere Beispiele zur Seite gestellt werden. Das Geschäft der Krankheitserfinder ist ein Megatrend in der modernen Medizin. Fünf verschiedene Strategien schälen sich heraus.

Erstens: Normale Prozesse des Lebens werden als medizinisches Problem verkauft. Vor den Männern waren schon die Frauen in die Wechseljahre gekommen. Diese gehen auch auf einen New Yorker Frauenarzt zurück, der vor einem halben Jahrhundert den Bestseller Feminine Forever (deutsch: Feminin für immer) veröffentlichte. Darin pries er aus dem Urin trächtiger Stuten hergestelltes Östrogen als Wundermittel für menopausale Frauen an. Erst viel später kam heraus, wer die Arbeit des Autors finanziell unterstützt hatte: das Östrogen-Unternehmen Wyeth Ayerst.

Zweitens: Persönliche und soziale Probleme werden als medizinisches Problem verkauft. Das Diagnostische und Statistische Manual Psychischer Störungen (DSM), ein Klassifikationssystem in der Psychiatrie, listet sämtliche Verhaltensweisen auf, die als krank gelten. Diese Bibel der Psychiatrie ist mit jeder Neuausgabe dicker geworden und erfasst inzwischen viele Verhaltensweisen, die bisher als normal galten.

An der «disruptiven Launenfehlregulationsstörung» oder DMDD (nach der englischen Wortschöpfung «Disruptive Mood Dysregulation Disorder») sollen Kinder leiden, die aufbrausen und Wutanfälle haben. Die von der US-Psychiaterin Ellen Leibenluft erfundene Störung sollen mehr als drei Prozent aller Kinder haben. So klein 
hat die in Aufmerksamkeitsdefizit-/Hyperaktivitätsstörung (ADHS) übrigens auch mal angefangen, heute zählt sie zu den häufigsten Kinderkrankheiten. Der Krankenkasse Barmer GEK zufolge bekamen knapp zwölf Prozent der Jungen im Alter von neun bis elf Jahren eine ADHSDiagnose gestellt.

Die Altersschusseligkeit hat es ebenfalls in den Rang einer quasi amtlichen psychiatrischen Krankheit geschafft und im neuen DSM den Namen «milde neurokognitive Störung» erhalten. Das «Trauerjahr» mag dem Volksmund geläufig sein, weil es die Leute als normal empfinden, wenn ein Mensch Zeit braucht, um den Verlust eines Angehörigen oder Freundes zu verwinden. Diese Frist war im alten DSM bereits auf zwei Monate begrenzt, nun wurde sie abermals verkürzt - auf 14 Tage. Die sogenannte Fressattacken-Störung (im englischen Original «Binge Eating Disorder») soll jene Menschen betreffen, die nicht immer kontrollieren können, wie viel oder wie schnell sie essen. Um als seelisch gestört zu gelten, reicht es, wenn diese Art der Nahrungsaufnahme einmal in der Woche vorkommt, und zwar über einen Zeitraum von drei Monaten.

Drittens: Risiken werden als Krankheit verkauft. Indem medizinische Fachgesellschaften willkürlich Grenzwerte festlegen, können sie über Nacht Millionen von Menschen in Patienten verwandeln. Den Grenzwert für Cholesterin haben Mediziner von 250 Milligramm pro Deziliter Blut auf 200 Milligramm verändert. Die Schwelle für Bluthochdruck senkten sie von 160 zu 100 auf 140 zu 90. Und die Mitglieder der privaten Medizinergruppe «Der alternde Mann» riefen einen Grenzwert zwölf Nanomol Testosteron pro Liter Blut aus - damit lagen ungefähr zwanzig Prozent der gesunden Durchschnittsmänner darunter. Einer der Grenzwertverschieber, ein Professor für Andrologie, trat später gegen Honorar auf Veranstaltungen der Industrie auf, um das TestosteronGel zu empfehlen.

Viertens: Seltene Symptome werden als grassierende Seuchen verkauft. Beispiel «erektile Dysfunktion»: Just zur Einführung der Potenzpille Viagra breitete sich die Impotenz in der Männerwelt erstaunlich aus. Auf einer Internetseite des Viagra-Herstellers Pfizer hiess es: «Erektionsstörungen sind eine ernstzunehmende und häufige Gesundheitsstörung: Ungefähr 50 Prozent der Männer zwischen dem 40. und 70. Lebensjahr sind davon betroffen - also jeder zweite.»

Freilich setzen Doktoren auf Gleichberechtigung und haben das Krankheitsbild der weiblichen Unlust entwickelt. Die «Hypoactive Sexual Desire Disorder» (HSDD) wurde auf einem Treffen von 19 Experten begründet, von denen 18 alle finanziell mit der Pharmaindustrie verbandelt waren. Laut einer Umfrage sollen 43 Prozent aller Frauen HSDD haben, aber auch dieser Befund wurde von einem Arzneimittelhersteller lanciert.

Die Mitarbeiter der Kampagne «Even the Score» (deutsch etwa: «Auch das Ergebnis») haben in den USA dafür die Zulassung des ersten Medikaments gegen HSDD getrommelt: Eine «pink Viagra» sei schon deshalb nötig, damit Männer mit der blauen Viagra nicht länger besser- gestellt seien. Tatsächlich hat die US-Arzneimittelbehörde das altbekannte Antidepressivum Flibanserin inzwischen gegen HSDD zugelassen. Die erfolgreiche Kampagne war vom Unternehmen Sprout Pharmaceuticals unterstützt worden, dem Hersteller des Flibanserins.

Fünftens: Leichte Symptome werden als Vorboten schwerer Leiden verkauft. Das Reizdarm-Syndrom etwa geht mit einer Fülle von Symptomen einher, die jeder schon einmal gespürt hat und viele als gewöhnliches Rumoren im Darm ansehen: Schmerzen, Durchfall und Blähungen.

Mit der Entwicklung einer Arznei erwachte das Interesse der Industrie an der angeblichen Krankheit. Das «British Medical Journal» zitierte aus einem heimlichen Entwurf einer PR- Firma. Ein auf drei Jahre angelegtes «medizinisches Erziehungsprogramm» sollte demnach den Reizdarm als "glaubhafte, häufige und richtige Krankheit» darstellen.

Das erklärte Ziel des Schulungsprogramms: «Das Reizdarm-Syndrom muss in den Köpfen der Doktoren als bedeutsamer und eigenständiger Krankheitszustand verankert werden.» Auch die Patienten «müssen überzeugt werden, dass das Reizdarmsyndrom eine weit verbreitete und anerkannte medizinische Störung ist».

In Wahrheit ist das Reizdarmsyndrom in weniger als fünf Prozent aller Fälle als wirklich ernst anzusehen. Einmal davon abgesehen, dass das Medikament, um das es damals ging, nicht besonders wirksam ist, wurde die Zulassung in Europa dann doch nicht beantragt. Offenbar war es dem Hersteller nicht gelungen, vorab einen Markt zu erschaffen. Es war nicht die einzige Pleite der Medikalisierer. Seitdem ich in meinem Buch Die Krankheitserfinder. Wie wir zu Patienten gemacht werden eine nach der Kaiserin Elisabeth von Österreich-Ungarn benannten, vermeintlich Form der Depression, das «Sisi-Syndrom», als trickreiche Hervorbringung der Industrie enthüllt habe, ist das Leiden von der Bildfläche verschwunden.

\section{The Disease Inventors}

The pharmaceutical industry is in the process of redefining human health with the aim of turning health into an unattainable state. Many ordinary processes of life - birth, age, sexuality, unhappiness and death - and normal behaviour patterns are systematically portrayed as pathological. Global corporations sponsor the invention of entire diseases and treatments, thereby generating new markets for their products. For there is much money to be made once you sedate fidgeting children, declare cholesterol the number one risk factor or introduce questionable screenings.

Keywords: Health, Pharmaceutical Industry, Disease Inventors, Medicalisation

\section{L'identificatore di malattie}

L'industria farmaceutica attualmente ridefinisce la salute umana in modo che la salute sia uno stato che non può essere mai raggiunto. Molti normali processi di vita - nascita, vecchiata, sessualità, infelicità e morte - che sono al di fuori dei 
normali modi di vita vengono rappresentati come patologici. Gruppi industriali operanti a livello globale sponsorizzano la scoperta di malattie e metodi di trattamento e immettono quindi i loro prodotti sul mercato. In questo modo, tenendo a bada bambini agitati, descrivendo la colesterina come il pericolo numero uno o prendendo misure preventive dubbie, è possibile guadagnare molto denaro.

Parole chiave: malattia, industria farmaceutica, identificatore di malattie, medicalizzazione

\section{Der Autor}

Seit seinem Enthüllungsbuch Die Krankheitserfinder - Wie wir zu Patienten gemacht werden hat sich der studierte
Biochemiker Jörg Blech (1966) als Autor etabliert, der den Dingen auf den Grund geht. Sein Buch löste eine europaweite Debatte über das Ausufern der Medizin aus und stand auf Platz 1 der deutschen Bestsellerliste. Das Schreiben hat Jörg Blech an der Henri-Nannen-Journalistenschule in Hamburg gelernt. Er war Wissenschaftsredakteur des Stern und der Zeit und ist nunmehr Mitglied der SPIEGEL-Redaktion. Seine Bestseller Heillose Medizin und Die Heilkraft der Bewegung sowie sein zum Klassiker gewordener Erstling Das Leben auf dem Menschen erscheinen bei Fischer Taschenbuch.

\section{Kontakt}

joerg.blech@spiegel.de 